\title{
Article \\ Significance of TERT Genetic Alterations and Telomere Length in Hepatocellular Carcinoma
}

\author{
Jeong-Won Jang ${ }^{1,2, *,+} \oplus$, Jin-Seoub Kim ${ }^{2,+}$, Hye-Seon Kim ${ }^{2}$, Kwon-Yong Tak ${ }^{1,2}$, Soon-Kyu Lee ${ }^{1,2}$ (D), \\ Hee-Chul Nam ${ }^{1,2}$, Pil-Soo Sung ${ }^{1,2} \mathbb{D}^{-}$, Chang-Min Kim ${ }^{3}$, Jin-Young Park ${ }^{3}$, Si-Hyun Bae ${ }^{1,2}$, Jong-Young Choi ${ }^{1,2}$ \\ and Seung-Kew Yoon ${ }^{1,2}$ (B)
}

1 Department of Internal Medicine, Collage of Medicine, The Catholic University of Korea, Seoul 06591, Korea; eworldcupzps@catholic.ac.kr (K.-Y.T.); blackiqq@catholic.ac.kr (S.-K.L.); hcnam@catholic.ac.kr (H.-C.N.); pssung@catholic.ac.kr (P.-S.S.); baesh@catholic.ac.kr (S.-H.B.); jychoi@catholic.ac.kr (J.-Y.C.); yoonsk@catholic.ac.kr (S.-K.Y.)

2 The Catholic University Liver Research Center, Department of Biomedicine \& Health Sciences, Collage of Medicine, The Catholic University of Korea, Seoul 06591, Korea; topiary@catholic.ac.kr (J.-S.K.); clover@catholic.ac.kr (H.-S.K.)

3 Department of Research \& Business Development, CbsBioscience Inc., Deajeon 34113, Korea; kcm3879@cbsbio.com (C.-M.K.); jonnypary@cbsbio.com (J.-Y.P.)

* Correspondence: garden@catholic.ac.kr; Tel.: +82-2-2258-6015; Fax: +82-2-3481-4025

+ Jeong-Won Jang and Jin-Seoub Kim contributed equally to this work.

check for updates

Citation: Jang, J.-W.; Kim, J.-S.; Kim, H.-S.; Tak, K.-Y.; Lee, S.-K.; Nam, H.-C.; Sung, P.-S.; Kim, C.-M.; Park, J.-Y.; Bae, S.-H.; et al. Significance of TERT Genetic Alterations and Telomere Length in Hepatocellular Carcinoma. Cancers 2021, 13, 2160. https://doi.org/10.3390/

cancers 13092160

Academic Editor: Lorenza Rimassa

Received: 20 April 2021

Accepted: 27 April 2021

Published: 30 April 2021

Publisher's Note: MDPI stays neutral with regard to jurisdictional claims in published maps and institutional affiliations.

Copyright: (c) 2021 by the authors. Licensee MDPI, Basel, Switzerland. This article is an open access article distributed under the terms and conditions of the Creative Commons Attribution (CC BY) license (https:// creativecommons.org/licenses/by/ $4.0 /)$.
Simple Summary: Telomerase reverse transcriptase (TERT) mutations are the most frequent genetic alterations in hepatocellular carcinoma (HCC). However, integrative analysis studies of TERT-telomere signaling during hepatocarcinogenesis are lacking. In this study, we investigated the clinicopathological association and prognostic value of TERT gene alterations and telomere length in HCC patients undergoing hepatectomy as well as transarterial chemotherapy (TACE). We found that there are eight key TERT-interacting genes and higher TERT expression and longer telomere length in HCC. We also found TERT-telomeric signals related to correlation with tumor differentiation and stage progression. TERT promoter mutations were an independent predictor of worse overall survival after hepatectomy, while TERT expression independently predicted worse time to progression after TACE. Telomere length was also associated with survival in TACE-treated patients. These findings suggest that TERT-telomere signals might be useful biomarkers for HCC, but the prognostic values may differ with tumor characteristics and treatment.

Abstract: Telomerase reverse transcriptase (TERT) mutations are reportedly the most frequent somatic genetic alterations in hepatocellular carcinoma (HCC). An integrative analysis of TERTtelomere signaling during hepatocarcinogenesis is lacking. This study aimed to investigate the clinicopathological association and prognostic value of TERT gene alterations and telomere length in HCC patients undergoing hepatectomy as well as transarterial chemotherapy (TACE). TERT promoter mutation, expression, and telomere length were analyzed by Sanger sequencing and real-time PCR in 305 tissue samples. Protein-protein interaction (PPI) analysis was performed to identify a set of genes that physically interact with TERT. The PPI analysis identified eight key TERT-interacting genes, namely CCT5, TUBA1B, mTOR, RPS6KB1, AKT1, WHAZ, YWHAQ, and TERT. Among these, $T E R T$ was the most strongly differentially expressed gene. TERT promoter mutations were more frequent, TERT expression was significantly higher, and telomere length was longer in tumors versus non-tumors. TERT promoter mutations were most frequent in HCV-related HCCs and less frequent in HBV-related HCCs. TERT promoter mutations were associated with higher TERT levels and longer telomere length and were an independent predictor of worse overall survival after hepatectomy. TERT expression was positively correlated with tumor differentiation and stage progression, and independently predicted shorter time to progression after TACE. The TERT-telomere network may have a crucial role in the development and progression of HCC. TERT-telomere abnormalities might serve as useful biomarkers for HCC, but the prognostic values may differ with tumor characteristics and treatment. 
Keywords: telomere; TERT; liver neoplasm; biomarkers; treatment outcome

\section{Introduction}

Hepatocellular carcinoma (HCC) is the most common primary liver cancer and the fifth leading cause of cancer-related mortality worldwide [1]. The major risk factors for HCC include liver cirrhosis, hepatitis B or C virus infection, alcohol abuse, nonalcoholic steatohepatitis, and metabolic disease. The mutation landscape in liver carcinogenesis is reportedly complicated, involving a number of pathways as well as somatic mutations in a multitude of genes [2-4]. Among the genetic alterations, telomerase reverse transcriptase (TERT) promoter mutations were reported to occur early and most frequently, affecting approximately $30-60 \%$ of all HCC patients [5,6]. Two hotspot mutations at -124 and -146 positions from the ATG start site in the TERT promoter have been shown to regulate TERT expression or the telomerase activation of human malignancies, including HCC [6,7].

Telomeres, repetitive DNA sequences (TTAGGG in vertebrates) found at the ends of the chromosome, are gradually shortened by each cell division in somatic cells, finally reaching senescence or apoptosis $[8,9]$. Telomeres are elongated by telomerase, a ribonucleoprotein-reverse transcriptase complex that uses its RNA as a template for the addition of simple telomeric repeats. Telomerase activity is regulated by the telomerebinding protein complex, called shelterin, which is composed of six proteins, including the telomeric repeat-binding factors (TRF) 1 and TRF2, the TRF1-interacting protein 2 (TIN2), protection of telomeres 1 (POT1), the POT1-TIN2 organizing protein (TPP1), and repressor/activator protein 1 (RAP1) [8]. However, embryonic stem cells and most cancer cells can maintain telomeres to overcome cell senescence or apoptosis. This process is controlled by TERT, the catalytic component of the telomerase complex that maintains telomere ends by addition of the telomere repeat TTAGGG [4,9]. Thus, TERT plays an important role in oncogenesis and the immortality of cancer cells.

Abnormalities in TERT expression or promoter mutations in HCC have been sporadically studied and reported to be associated with cancer recurrence and progression [10-14]. However, the clinical implications and molecular mechanisms underlying HCC initiation and progression are still unclear because only limited studies have been conducted and the previous studies included only a small number of patients or were limited only to the surgical setting of early-stage HCC. Thus, there is insufficient knowledge about how and what changes in TERT occur from early- to late-stage HCC. Moreover, no studies have attempted to evaluate the impact of the TERT-telomere network and alterations on the outcomes of various stages of HCC treated by surgical and non-surgical options.

To address these issues, the present study correlated the genetic alterations in TERT, gene expression, and telomere length with the clinicopathological features of HCC. In addition, the potential roles of the TERT-telomere network as a prognostic biomarker within the setting of hepatectomy and transarterial chemoembolization (TACE) were evaluated and compared.

\section{Results}

\subsection{Baseline Characteristics}

A total of 305 liver tissue samples were obtained from the 205 patients, including 105 tumor tissues only and 100 paired tumor and non-tumor tissues. The median patient age was 60 years old, and 165 patients $(80.5 \%)$ were male. The causes of liver disease included hepatitis B virus $(n=138,67.3 \%)$, hepatitis $C$ virus $(n=16 ; 7.8 \%)$, and other non-viral diseases $(n=51 ; 24.9 \%)$. Most patients had Child-Pugh class A hepatic function $(n=167 ; 81.5 \%)$. The mean tumor size was $6.5 \pm 4.9 \mathrm{~cm}$ and 94 (45.9\%) patients had multiple tumors. The patients in the surgical group were younger and had less advanced HCC compared to those in the TACE group. The baseline characteristics of the patients are shown in Table 1. 
Table 1. Baseline characteristics of the study population.

\begin{tabular}{|c|c|c|c|}
\hline Characteristics & $\begin{array}{l}\text { All Patients } \\
\quad(n=205)\end{array}$ & $\begin{array}{l}\text { Surgical Group } \\
\quad(n=121)\end{array}$ & $\begin{array}{l}\text { TACE Group } \\
\quad(n=84)\end{array}$ \\
\hline \multicolumn{4}{|l|}{ Sex } \\
\hline Male & $165(80.5)$ & $89(73.5)$ & $76(90.5)$ \\
\hline Female & $40(19.5)$ & $32(26.4)$ & $8(9.5)$ \\
\hline Age (years) & $60.0 \pm 11.7$ & $58.1 \pm 11.6$ & $64.5 \pm 11.9$ \\
\hline \multicolumn{4}{|l|}{ Cause of liver disease } \\
\hline $\mathrm{HBV}$ & $138(67.3)$ & $85(70.2)$ & $53(63.1)$ \\
\hline $\mathrm{HCV}$ & $16(7.8)$ & $13(10.7)$ & $3(3.6)$ \\
\hline Non-viral & $51(24.9)$ & $23(19.0)$ & $28(33.3)$ \\
\hline AST (IU/L) & $45(29.5-94.5)$ & $45(30-94.3)$ & $42(29-91)$ \\
\hline ALT (IU/L) & $34(23-68.5)$ & $33.5(23-69)$ & $33(22-65)$ \\
\hline \multicolumn{4}{|l|}{ Child-Pugh class } \\
\hline $\mathrm{A}$ & $167(81.5)$ & $111(91.7)$ & $56(66.7)$ \\
\hline $\mathrm{B} / \mathrm{C}$ & $38(18.5)$ & $10(8.3)$ & $28(33.3)$ \\
\hline Tumor size $(\mathrm{cm})$ & $6.5 \pm 4.9$ & $4.9 \pm 4.5$ & $8.8 \pm 4.9$ \\
\hline \multicolumn{4}{|l|}{ Tumor number } \\
\hline Single & $111(54.1)$ & $88(72.7)$ & $23(27.4)$ \\
\hline Multifocal & $94(45.9)$ & $33(27.3)$ & $61(72.6)$ \\
\hline$\alpha$-fetoprotein (ng/mL) & $50.3(5.5-800.8)$ & $48.4(5.4-699.1)$ & $57.4(5.6-881.4)$ \\
\hline \multicolumn{4}{|l|}{ mUICC stage } \\
\hline $\mathrm{I}$ & $13(6.3)$ & $9(7.4)$ & $4(4.8)$ \\
\hline II & $80(39.0)$ & $66(54.5)$ & $14(16.7)$ \\
\hline III & $49(23.9)$ & $29(24.0)$ & $20(23.8)$ \\
\hline IV & $63(30.7)$ & $17(14.0)$ & $46(54.8)$ \\
\hline
\end{tabular}

HBV, hepatitis B virus; HCV, hepatitis C virus; AST, aspartate aminotransferase; ALT, alanine aminotransferase; mUICC, modified Union for International Cancer Control. Data are expressed as mean $\pm \mathrm{SD}$ or median (interquartile range). Figures in parentheses indicate percentage.

\subsection{Protein-Protein Interaction with TERT Gene Sets and Gene Expression}

The protein-protein interaction (PPI) analysis was performed through CBS probe PINGS $^{\text {TM }}$ based on the STRING database to establish a set of genes interacting with TERT. Functional clustering of the PPI analysis identified eight genes, including TERT, AKT1, CCT5, $m$ TOR, RPS6KB1, TUBA1B, YWHAQ, and YWHAZ, as protein complexes related to TERT (Figure 1A). Functional interactions between the eight proteins are summarized in Table S1. Within the eight genes, we performed gene expression analysis in the tumor and non-tumor paired samples. TERT mRNA was significantly overexpressed in the tumors compared to the non-tumors $(p<0.001)$, whereas the expression levels of the other seven genes were not significantly different between the tumors and non-tumors (Figure 1B).

A

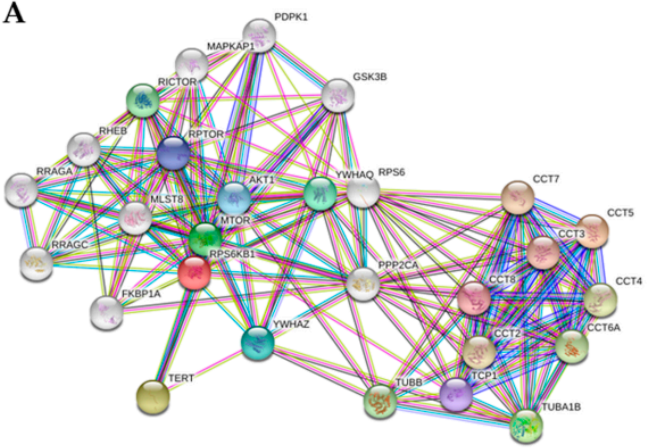

Colored nodes: proteins and first shell of interactors White nodes: second shell of interactors
B

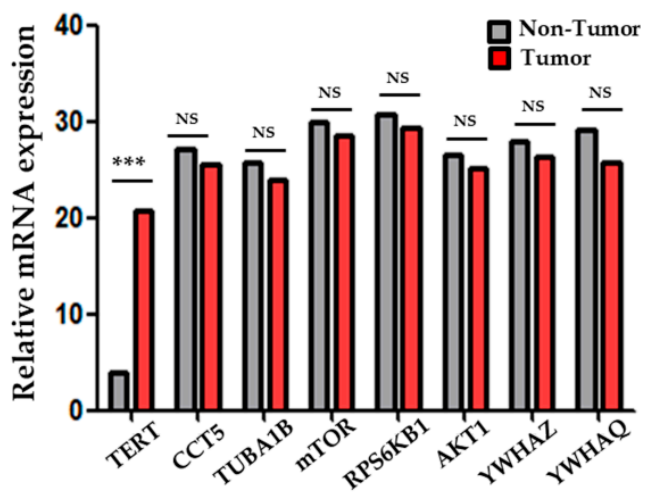

Figure 1. Cont. 


\section{C}
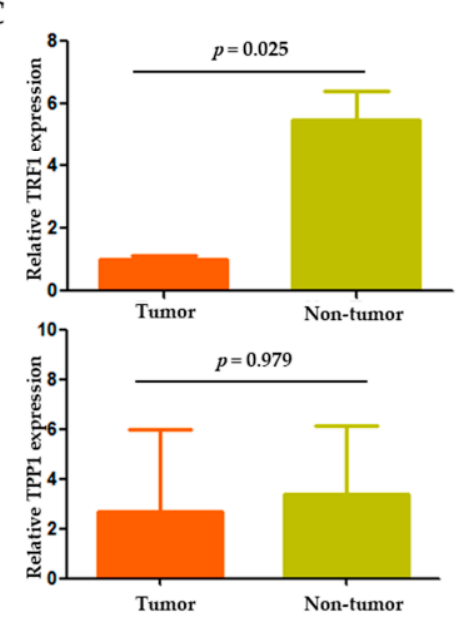

D

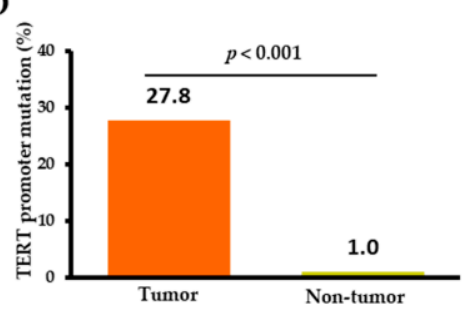

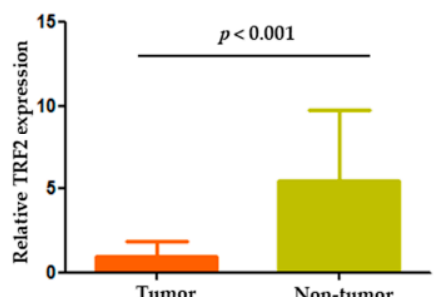
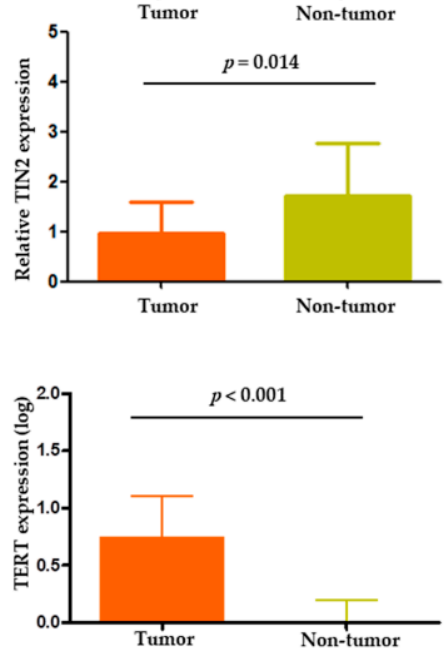
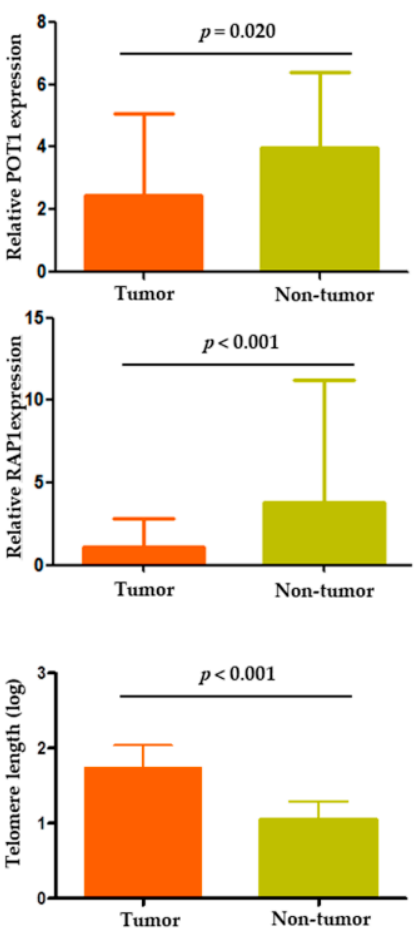

Figure 1. TERT expression and telomere length relationship in HCC. (A) Protein-protein interaction (PPI) analysis of TERT using the CBS probe PINGS ${ }^{T M}$ in HCC tissues. (B) Expression profiles of the eight TERT-interacting genes from the STRING database in tumor versus adjacent non-tumor tissues. (C) Comparison of shelterin complex TRF1, TRF2, POT1, TPP1, TINP2, and RAP1 between tumor and non-tumor tissues. (D) Comparison of TERT promoter mutations, TERT expression and telomere length between tumor and non-tumor tissues. TRF, telomeric repeat-binding factors; POT1, protection of telomeres 1; TPP1, POT1-TIN2 organizing protein; TIN2, TRF1 and TRF2 interacting nuclear protein 2; RAP1, repressor/activator protein 1 . NS, non-significance; ${ }^{* * *} p<0.001$.

Besides the genes derived from the PPI analysis, we also analyzed the shelterin complex which is known to regulate telomerase activity [8]. As a result, all shelterin components (TRF1, TRF2, POT1, TIN2, and RAP1) except TPP1 were significantly overexpressed in the non-tumors compared to the tumors $(p<0.001$; Figure $1 C)$. Negative correlations were seen between the expression statuses of the shelterin complex proteins, TERT, and telomere length (Table S2 and Figure S1).

\subsection{Comparison of TERT and Telomere Length in Tumors versus Non-Tumors}

Overall, the TERT gene and its function were markedly altered in the tumors compared to the adjacent non-tumor samples. Two hotspot mutations in the TERT promoter region, $-124 \mathrm{C}>\mathrm{T}(\mathrm{C} 228 \mathrm{~T})$ and $-146 \mathrm{C}>\mathrm{T}(\mathrm{C} 250 \mathrm{~T})$, were observed in 57 (27.8\%) HCC samples but in only one $(1.0 \%)$ non-tumor sample $(p<0.001$; Figure 1D). Telomere length were assessed in 86 evaluable samples. The absolute telomere length was $39.8 \pm 2.9 \mathrm{~kb}$ (interquartile range: $10.79-110.41$ ), with $55.9 \pm 4.1 \mathrm{~kb}$ for the tumor and $8.8 \pm 0.6 \mathrm{~kb}$ for the nontumor samples. The TERT expression and telomere length were significantly higher and longer, respectively, in the tumors than in the adjacent non-tumor tissues (all $p<0.001$; Figure 1D). These findings indicate an important role of TERT and telomere dysfunction in hepatocarcinogenesis.

\subsection{Alterations in TERT and Telomere Length in Relation to Clinical and Tumor Characteristics}

The 57 tumoral TERT promoter mutations included $54(94.7 \%)$ with $-124 \mathrm{C}>\mathrm{T}$ and 3 (5.3\%) with $-146 \mathrm{C}>\mathrm{T}$ mutations. These two mutations were mutually exclusive in HCC. The prevalence of TERT promoter mutations differed according to the etiology of liver disease. The 57 patients with the mutation consisted of 32 with HBV-related HCC (23.2\%), 
7 with HCV-related HCC (43.8\%), and 18 with NBNC (non-HBV and non-HCV) (35.3\%), showing that the mutation rates were highest in HCV-related HCCs and lowest in HBVrelated HCCs (Figure 2A). In the tumors, the presence of TERT promoter mutation may be associated with an increasing level of TERT expression and telomere length (Figure 2B), which suggests that tumoral TERT expression and telomere length were affected by the TERT promoter mutation status.

A

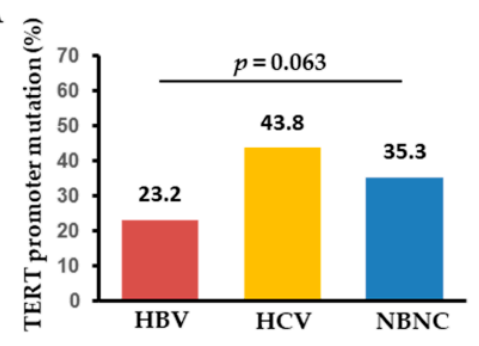

$\mathrm{C}$

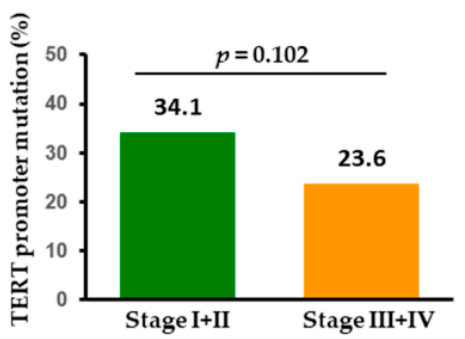

D

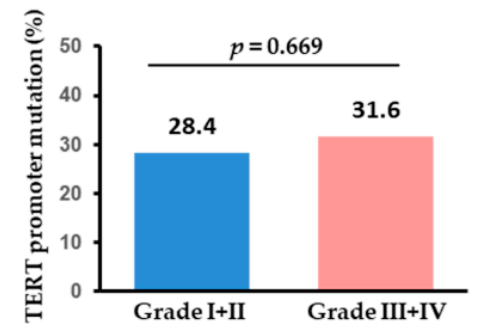

B
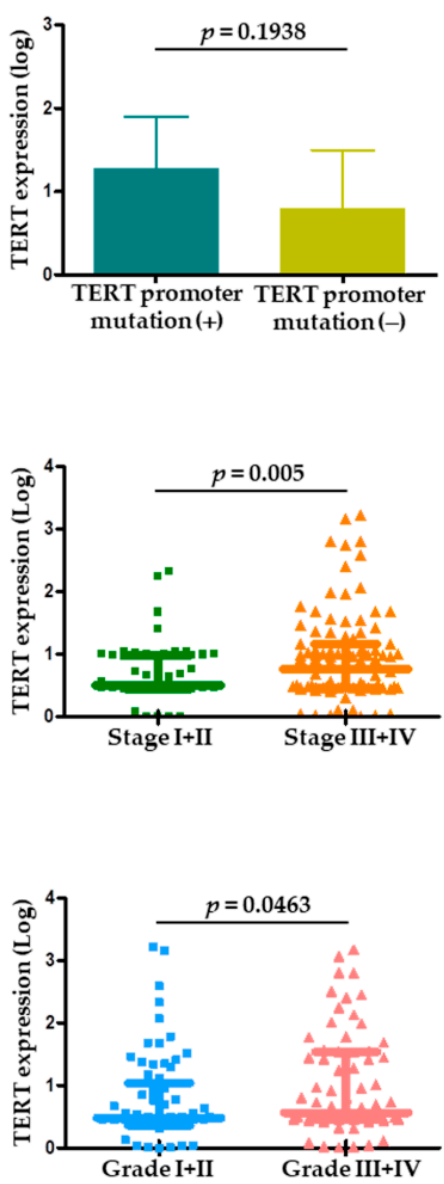
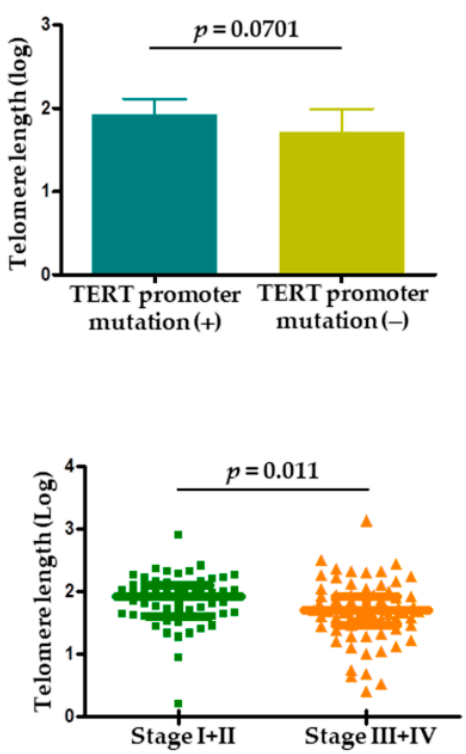

mutation(+) mutation(-)

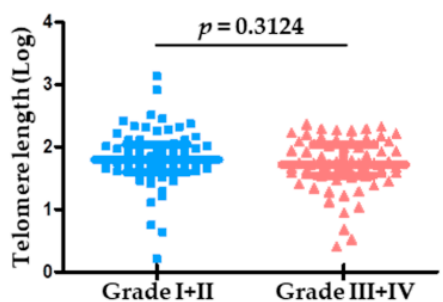

Figure 2. (A) Frequency of TERT promoter mutations in HCC according to the etiology of HCC. (B) TERT expression and telomere length in the presence or absence of TERT promoter mutations in HCC. Frequency of TERT promoter mutations, TERT expression, and telomere length according to (C) tumor stage and (D) tumor histological grade. HBV, hepatitis B virus; $\mathrm{HCV}$, hepatitis $\mathrm{C}$ virus; NBNC, non-HBV non-HCV.

When analyzed by tumor characteristics, TERT promoter mutations tended to be more frequent in single $(37 / 109,33.9 \%)$ versus multiple $(20 / 92,21.7 \%)$ HCC $(p=0.056)$. With tumor stage progression, the TERT level was significantly upregulated $(p=0.005)$, whereas telomere length significantly decreased $(p=0.011)$ (Figure 2C). Regarding pathological tumor differentiation, TERT expression levels were positively correlated with HCC histological grade, while TERT promoter mutations or telomere length were not significantly different between the grades (Figure 2D).

\subsection{TERT Abnormalities and Outcome after Hepatectomy}

Patients with TERT promoter mutations had significantly worse overall survival (OS) than those without $(p=0.024$; Figure 3A). Together with tumor extent, TERT promoter mutations (hazard ratio (HR) $=4.24,95 \%$ CI: $1.75-10.26, p=0.001$ ) were identified as an independent factor for OS after hepatectomy (Table 2). Regarding post-operative recurrence, patients with TERT promoter mutations tended to suffer from earlier cancer 
recurrence than those without, but the difference did not reach statistical significance $(p=0.159 ;$ Figure 3B). Interestingly, although not statistically significant in the univariate analysis, TERT promoter mutations ( $\mathrm{HR}=2.98,95 \% \mathrm{CI}: 1.01-8.33, p=0.048$ ) were finally identified as an independent factor for recurrence after hepatectomy in the multivariate analysis (Table 2). The prognostic performance of TERT mutations was better in patients with non-HBV-related HCC than in those with HBV-related HCC (Figure S2). Unlike TERT mutations, TERT expression levels or telomere length had no significant impacts on OS or recurrence after hepatectomy (Figure 3C-F).

A

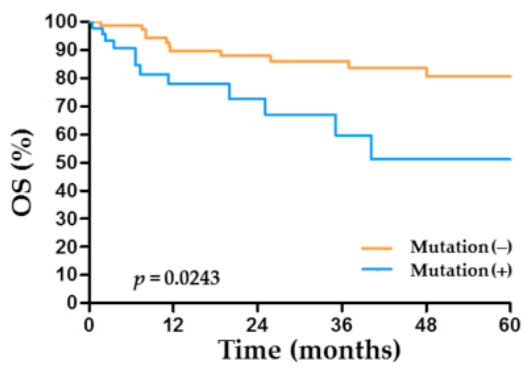

C

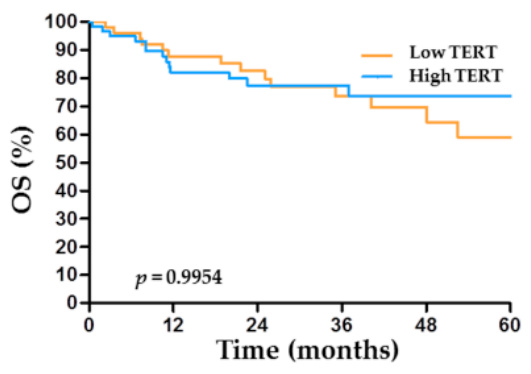

E

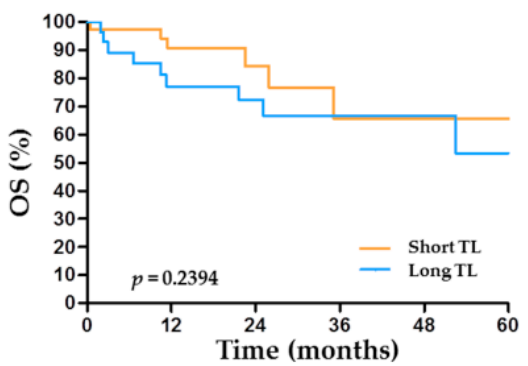

B

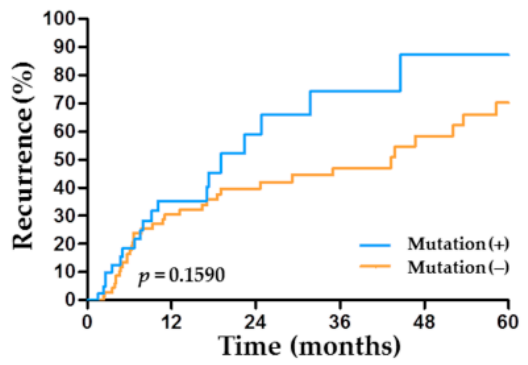

D

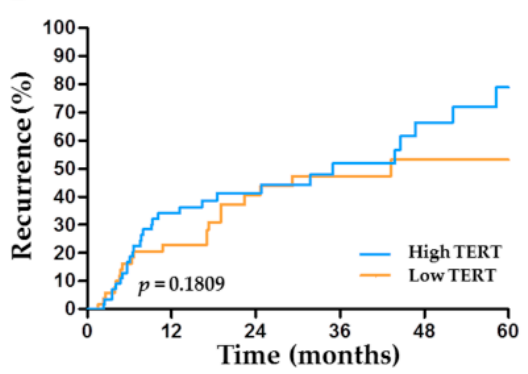

F

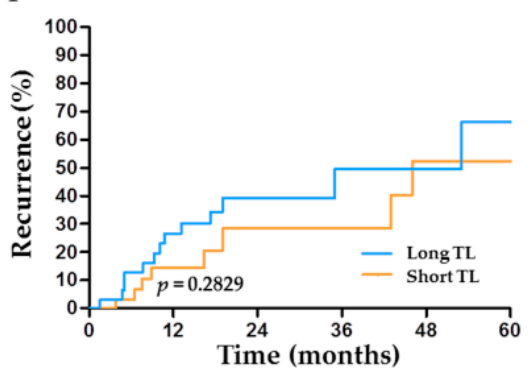

Figure 3. Prognostic role of TERT factors in the outcome of HCC after hepatectomy. Overall survival (OS) and HCC recurrence according to $(\mathbf{A}, \mathbf{B})$ the status of TERT promoter mutations, $(\mathbf{C}, \mathbf{D})$ the expression level of TERT, and $(\mathbf{E}, \mathbf{F})$ telomere length.

\subsection{TERT Abnormalities and Outcome after TACE}

The clinical outcomes according to TERT factors in patients undergoing TACE are depicted in Figure 4A-F. High TERT levels were associated with significantly shorter time to progression (TTP) $(p=0.0081)$ in the TACE group (Figure 4D). Together with the tumor stage, high levels of TERT expression (HR $=2.06,95 \%$ CI: $1.06-4.01, p=0.033$ ) were identified as an independent predictor of shorter TTP after TACE (Table 3). Unlike its predictive value after hepatectomy, the status of TERT promoter mutations had no impact on OS or TTP after TACE (Figure $4 \mathrm{~A}, \mathrm{~B}$ ). 
Table 2. Prognostic variables in patients undergoing hepatic resection.

\begin{tabular}{|c|c|c|c|c|c|c|}
\hline \multirow{3}{*}{ Variables } & \multicolumn{3}{|c|}{ Overall Survival } & \multicolumn{3}{|c|}{ Recurrence } \\
\hline & Univariate & Multivariate & & Univariate & Multivariate & \\
\hline & $p$ & HR $(95 \%$ CI $)$ & $p$ & $p$ & HR (95\% CI) & $p$ \\
\hline Male sex & 0.423 & & & 0.694 & & \\
\hline Age $>60$ years & 0.319 & & & 0.047 & $5.07(1.67-15.15)$ & 0.004 \\
\hline Cause of liver disease & 0.133 & & & 0.144 & & \\
\hline $\mathrm{AST}>90 \mathrm{U} / \mathrm{L}$ & 0.353 & & & 0.473 & & \\
\hline $\mathrm{ALT}>60 \mathrm{U} / \mathrm{L}$ & 0.357 & & & 0.595 & & \\
\hline Child-Pugh class B/C & 0.095 & $1.02(0.94-1.09)$ & 0.616 & 0.120 & & \\
\hline Tumor size $>5 \mathrm{~cm}$ & $<0.001$ & $7.97(3.13-20.24)$ & $<0.001$ & $<0.001$ & $1.81(0.63-5.18)$ & 0.263 \\
\hline Tumor multiplicity & 0.075 & $1.56(0.43-5.65)$ & 0.491 & 0.019 & $1.85(0.44-7.71)$ & 0.398 \\
\hline$\alpha$-fetoprotein & 0.317 & & & $<0.001$ & $1.54(0.56-4.23)$ & 0.396 \\
\hline Tumor stage (mUICC) & $<0.001$ & $2.46(1.45-4.16)$ & 0.001 & $<0.001$ & $2.71(1.48-4.96)$ & 0.001 \\
\hline TERT promoter mutation & 0.029 & $4.24(1.75-10.26)$ & 0.001 & 0.162 & $2.98(1.01-8.33)$ & 0.048 \\
\hline TERT expression & 0.913 & & & 0.362 & & \\
\hline Telomere length & 0.446 & & & 0.208 & & \\
\hline
\end{tabular}

$\mathrm{HR}$, hazard ratio; $\mathrm{CI}$, confidence interval; $\mathrm{HBV}$, hepatitis B virus; $\mathrm{HCV}$, hepatitis $\mathrm{C}$ virus; $\mathrm{AST}$, aspartate aminotransferase; $\mathrm{ALT}$, alanine aminotransferase; mUICC, modified Union for International Cancer Control; TERT, telomerase reverse transcriptase.

A

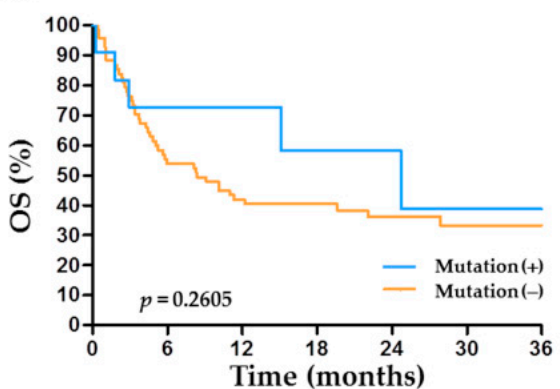

C

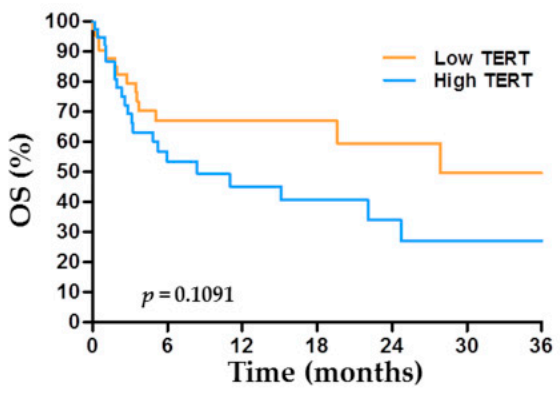

E

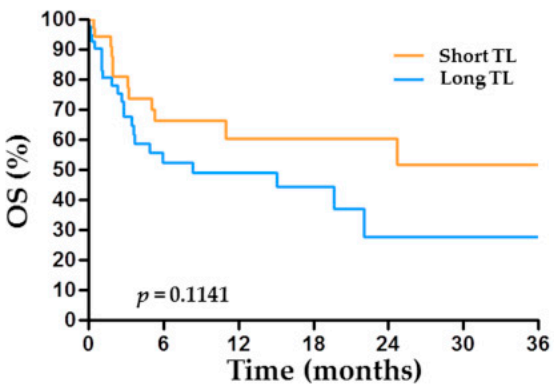

B

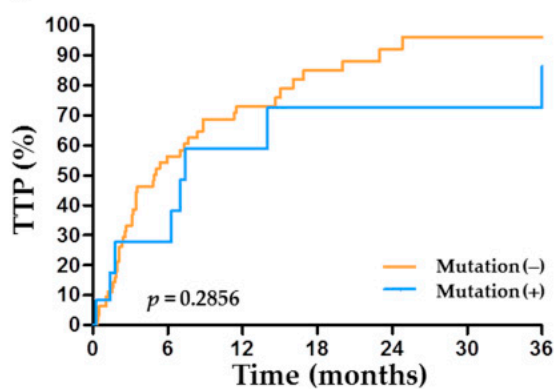

D

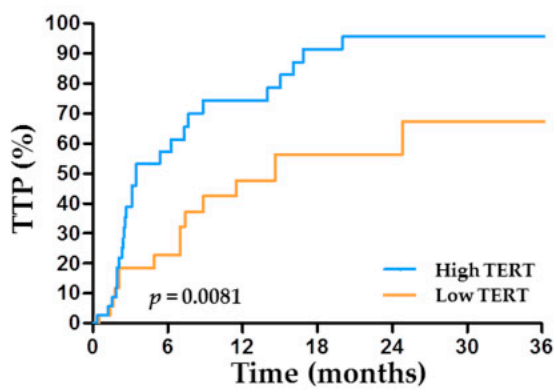

$\mathbf{F}$

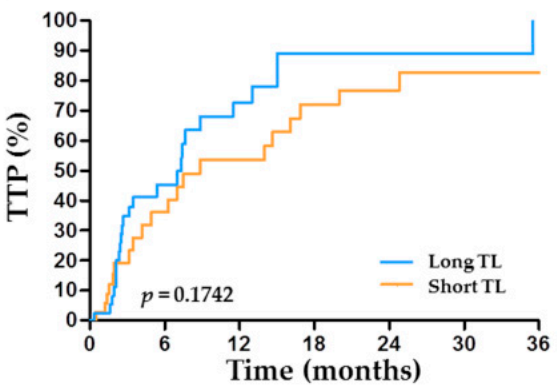

Figure 4. Prognostic role of TERT factors in the outcome of HCC after transarterial chemoembolization (TACE). Overall survival (OS) and time to progression (TTP) according to (A,B) the status of TERT promoter mutations, $(\mathbf{C}, \mathbf{D})$ the expression level of TERT, and (E,F) telomere length (TL). 
Table 3. Prognostic variables in patients undergoing TACE-based treatment.

\begin{tabular}{|c|c|c|c|c|c|c|}
\hline \multirow{3}{*}{ Variables } & \multicolumn{3}{|c|}{ Overall Survival } & \multicolumn{3}{|c|}{ Time to Progression } \\
\hline & Univariate & Multivariate & & Univariate & Multivariate & \\
\hline & $p$ & HR $(95 \%$ CI $)$ & $p$ & $p$ & HR (95\% CI) & $p$ \\
\hline Male sex & 0.420 & & & 0.958 & & \\
\hline Age $>60$ years & 0.575 & & & 0.800 & & \\
\hline Cause of liver disease & 0.150 & & & 0.291 & & \\
\hline $\mathrm{AST}>88 \mathrm{U} / \mathrm{L}$ & 0.042 & $1.55(0.49-3.12)$ & 0.644 & 0.067 & $1.44(0.72-2.90)$ & 0.300 \\
\hline ALT > $57 \mathrm{U} / \mathrm{L}$ & 0.119 & & & 0.283 & & \\
\hline Child-Pugh class B/C & $<0.001$ & $4.55(1.96-10.55)$ & 0.001 & 0.087 & $1.27(0.54-2.96)$ & 0.576 \\
\hline Tumor size $>5 \mathrm{~cm}$ & 0.001 & $2.68(0.83-8.67)$ & 0.099 & 0.003 & $2.11(0.96-4.62)$ & 0.061 \\
\hline Tumor multiplicity & 0.469 & & & 0.102 & & \\
\hline$\alpha$-fetoprotein & 0.003 & $2.67(1.01-7.05)$ & 0.047 & 0.061 & $1.07(0.51-2.23)$ & 0.855 \\
\hline Tumor stage (mUICC) & $<0.001$ & $1.98(1.02-3.84)$ & 0.043 & $<0.001$ & $1.70(1.11-2.60)$ & 0.013 \\
\hline TERT promoter mutation & 0.181 & & & 0.330 & & \\
\hline TERT expression & 0.028 & $1.58(0.67-3.70)$ & 0.289 & 0.010 & $2.06(1.06-4.01)$ & 0.033 \\
\hline Telomere length & 0.137 & & & 0.277 & & \\
\hline
\end{tabular}

TACE, transarterial chemoembolization; HR, hazard ratio; CI, confidence interval; HBV, hepatitis B virus; HCV, hepatitis C virus; $\mathrm{AST}$, aspartate aminotransferase; ALT, alanine aminotransferase; mUICC, modified Union for International Cancer Control; TERT, telomerase reverse transcriptase.

\section{Discussion}

The current study, involving a large cohort of patients, evaluated the clinicopathological association of TERT abnormalities and their biomarker functions in predicting HCC outcomes. In our results, the clinicopathological association and patterns of the TERT-telomere network varied substantially with the underlying disease, tumor stage, and differentiation. The prevalence of TERT promoter mutations was associated with significantly poorer OS and a trend toward an increased risk of HCC recurrence after hepatic resection. In addition, higher TERT expression was associated with advancing tumor stage and cell differentiation and shorter TTP and OS after TACE. Telomere length was correlated with TERT alterations and tumor characteristics. The overall findings suggest the crucial oncogenic role of TERT-telomere abnormalities and their utility as a prognostic factor for the outcome of HCC patients. To the best of our knowledge, our study was the first to provide a comprehensive analysis of the TERT-telomere network in settings of both surgical and non-surgical treatments of HCC.

In our analysis, TERT was the strongest differentially expressed gene among the TERT-interacting genes identified by the PPI analysis. All of the TERT-related markers such as TERT expression, promoter mutations, and telomere length were markedly enhanced in the tumors versus the adjacent non-tumorous tissues. In contrast, the expression of shelterin components was significantly reduced in the tumors compared to the non-tumors, indicating that the shelterin complex functions as a negative regulator of telomerase [8]. These findings again underline the fundamental role of TERT and telomere biology in hepatocarcinogenesis.

An interesting finding of our study was that the predictive role of TERT promoter mutations appeared to differ with treatment, with apparent effects on the post-surgical outcomes but not on those of non-surgical treatment. Although TERT promoter mutations have been found in preneoplastic lesions or early-stage HCCs as a gatekeeper event [15,16], its frequency reportedly did not appear to further increase linearly with disease progression after the establishment of HCC. In a study examining hepatic nodules development in cirrhosis, the hotspot TERT promoter mutations were detected in $6 \%$ of the low-grade dysplastic nodules, $19 \%$ of the high-grade dysplastic nodules, $61 \%$ of early HCC, and $41 \%$ of established HCC [15]. Indeed, our study showed a slightly decreasing trend in the frequency of promoter mutations from early- to advanced-stage HCC and a poor correlation with the outcome of patients with later-stage HCC eligible for TACE. Thus, it is presumed that the biomarker function of TERT mutations might be more apparent in 
early-stage HCC but was gradually outweighed by the tumor-promoting effects of other concomitant driver mutations emerging during HCC progression.

The prevalence of TERT promoter mutations was significantly different according to the underlying liver disease, with the highest frequency in HCV-related HCC (44\%) followed by non-viral (38\%) and HBV-related HCC (23\%). The mutation rates were largely consistent with those in previous studies, which reported mutation frequencies of $50-60 \%$ in HCV-related and 25-35\% in HBV-related HCCs [9-11]. Interestingly, we observed the more apparent association of TERT mutations with the prognosis of non-HBV HCC rather than HBV-related HCCs in the surgical group (Figure S2). However, due to the insufficient number of patients in each subgroup, the interesting issues of whether the prognostic performance of TERT mutations may vary with tumor stage, treatment, and/or the cause of HCC should be confirmed in larger studies.

Another TERT marker, TERT gene expression, was significantly associated with worse prognosis after TACE but not after surgical resection. The observation that higher TERT expression levels were an independent factor predicting shorter TTP suggests the tumorpromoting effects of TERT reactivation [17]. Of note, there was no significant association between TERT promoter mutations and TERT expression in our data (Figure 2B). Nevertheless, TERT expression became significantly enhanced with the progression of tumor stage and tissue differentiation, which was eventually linked to tumor progression in TACE-treated patients. TERT mutations were reported to correlate with tumor initiation, whereas other mutations, such as those in TP53 or CTNNB1, were associated with later stages of HCC, causing further genomic modifications [15]. Since, in our study, advancedstage HCC harbored fewer TERT mutations compared to early-stage HCC, the higher TERT expression observed in advanced HCC might be a consequence of other telomerasereactivating mechanisms, including HBV integration into the TERT sequence, TERT gene amplification, or the accumulation of oncogenic pathways with tumor growth, which are distinct from the mechanisms of TERT promoter mutations [6,17]. Taken together, the overall findings on TERT alterations imply that TERT promoter mutations would better predict the outcome of patients with early-stage HCC treated with surgery, whereas TERT expression may be more associated with the prognosis of later-stage HCC patients eligible for non-surgical treatments.

Together with TERT genetic alterations, telomere length was significantly longer in tumors versus non-tumors. Intriguingly, telomere length was shorter with tumor stage progression (Figure 2C), indicating that moderate genomic instability elicited by shortened telomeres might be advantageous to cancer evolution in advanced HCC $[8,18]$. Moreover, despite the absence of a correlation between relative telomere length and postsurgical outcomes, telomere length appeared potentially predictive of outcomes within the TACE-treated patients (Figure 4E,F). Our results are inconsistent with a study showing an association between telomere length and survival after hepatectomy, but consistent in that the study results suggest the potential prognostic role of telomere length in a subset of HCC patients [11]. Telomere length is under the control of the telomerase and shelterin complex. It was reported that some shelterin components were associated with HCC expressing stemness markers and that their expression was dependent on the cause of liver disease $[19,20]$. However, very limited information is available about the impact of shelterin complex on the outcome of HCC. The regulation of telomere length in relation to shelterin and TERT alterations in HCC also remains largely unknown and requires further investigation.

Our study had several limitations. It was a retrospective analysis and inevitably subject to selection bias. Thus, our findings need to be validated in large-scale prospective cohort studies. The majority (68\%) of our patients had HBV-related HCC, with fewer TERT mutations compared to other etiologies. Thus, our data should be evaluated in different ethnic groups for further generalization. The TACE group was heterogeneous in HCC stages and not limited to intermediate HCC but also included advanced HCC patients, for which other treatments are currently indicated. The function of shelterin 
complex was incompletely studied regarding the protein expression and its prognostic implication in HCC. Nevertheless, the current study represented an integrative analysis of TERT-related factors, including TERT expression, promoter mutations, and telomere length across patients undergoing not only surgical resection but also non-surgical treatments and, thus, provides a more comprehensive understanding of the entire scope of TERT-telomere biology in hepatocarcinogenesis.

\section{Materials and Methods}

\subsection{Patients and Treatment}

A total of 205 patients who were diagnosed with HCC at the Catholic University of Korea and the Catholic Central Biobank between March 2011 to February 2019 were analyzed. HCC was diagnosed based on histological evidence, $\alpha$-fetoprotein levels, or typical radiological findings according to the Korean National Cancer Center (KNCC) guidelines [21]. Histological grading of HCC was performed using the Edmonson and Steiner grading scheme [22]. Tumor stage was classified according to the modified Union for International Cancer Control (mUICC) stage endorsed by the KNCC guidelines [21]. Treatment for HCC was performed based on tumor stage and liver function according to the KNCC practice guidelines [21]. Briefly, the patients were offered surgical resection if their tumors were resectable and they had acceptable liver function. TACE using doxorubicin was offered to patients who had unresectable or multifocal HCCs. Anticancer treatments were categorized into surgical (hepatectomy) and non-surgical options (TACE-based treatments). Treatment for recurrent or refractory tumors after initial therapy was decided by multidisciplinary decision-making and the KNCC guidelines [21]. This study was approved by the Ethics Committees of The Catholic University of Korea and all other participating institutions in accordance with the 1975 Declaration of Helsinki. The patients provided informed consent to participate in the study.

\subsection{Protein-Protein Interaction Methods}

Protein-protein interactions were analyzed using CBS probe PINGS ${ }^{\mathrm{TM}}$ (Protein Interaction Network Generation System, KR100957386B1; Daejon, Korea) to identify the genes interacting with TERT. CBS probe PINGS ${ }^{\mathrm{TM}}$ uses five modules (protein-protein interactions, Path-finder, Path-linker, Path-maker, and Path-lister) to identify interacting genes, interaction distance, and interaction frequency [23].

A multi-functional analytical tool, CBS Probe PINGS ${ }^{\mathrm{TM}}$, was used to match TERT gene with its Entrez Gene record (NCBI ID, https: / www.ncbi.nlm.nih.gov/gene, accessed on 17 November 2017) from the iProClass (https://www.ncbi.nlm.nih.gov/pubmed/ 15022647, accessed on 17 November 2017) database, and with gene names and synonyms in UniProtKB/Swiss-Prot (Uniprot Knowledgebase, https: / / www.ncbi.nlm.nih. gov/pubmed/27899622, accessed on 17 November 2017) to further interchange with identification factor "Uniprot Ac" in CBS Probe PINGS ${ }^{\mathrm{TM}}$. We then conducted the interactive proteins network analysis leveraging IntAct (IntAct, http:/ / europepmc.org/ abstract/MED/24234451, accessed on 17 November 2017), BioGRID (Biological General Repository for Interaction Datasets, https: / / www.ncbi.nlm.nih.gov/pubmed/30476227, accessed on 17 November 2017), DIP (Database of interacting proteins, https:/ / www.ncbi. nlm.nih.gov / pubmed/10592249, accessed on 17 November 2017), HPRD (Human Protein Reference Database, https:/ / www.ncbi.nlm.nih.gov/pubmed/18988627, accessed on 17 November 2017), and MINT (The Molecular INTeraction, https: / www.ncbi.nlm.nih.gov / pmc/articles/PMC1751541, accessed on 17 November 2017) database accordingly. The selectable identification includes interaction distance, interaction type, interaction detection method, number of interactive information-related databases, number of related literature studies, and number of interaction detection methods. In this study, we investigated the direct interacting genes with the start gene as TERT, and the organism has been chosen as Homo sapiens. 


\subsection{TERT Promoter Mutation}

Genomic DNA (gDNA) was extracted from fresh frozen tissue samples using a QIAamp DNA Mini Kit (Qiagen, Hidden, Germany). Direct sequencing of the tissue samples was performed for polymerase chain reaction (PCR) amplification using the following pairs of primers encompassing the mutational hotspots. For $-124 \mathrm{bp} \mathrm{G>A}$ and $-146 \mathrm{bp}$ $\mathrm{G}>\mathrm{A}$ in the TERT promoter, the primers were forward 5'-CAGCGCTGCCTGAAACTC-3' and reverse $5^{\prime}$-GTCCTGCCCCTTCACCTT- $3^{\prime}$. PCR was performed using a DNA Engine Tetrad 2 Peltier Thermal Cycler (Bio-Rad, Hercules, CA, USA). The sequencing data were analyzed on an ABI PRISM 3730XL Analyzer (Applied Biosystems, Foster City, CA, USA).

\subsection{Quantitative Real-Time PCR Analysis of TERT Gene Expression and Shelterin Complex}

Total RNA was extracted from $25 \mathrm{mg}$ of fresh frozen tissue sample using a miRNeasy Mini Kit (Qiagen) according to the manufacturer's instructions. TERT gene expression was measured by quantitative real-time PCR (qRT-PCR) using the Hs00972650_m1 TaqMan gene expression assay (Applied Biosystems, Foster City, CA, USA). The primers and probe sequences of TERT-interacting genes (CCT5, TUBA1B, mTOR, RPS6KB1, AKT1, YWHAZ, and $Y W H A Q$ ) were designed in Primer Express 3.0 (Applied Biosystems). All probes were labeled with TAMRA at the $3^{\prime}$ end and FAM at the $5^{\prime}$ end (Table S3). All measurements were normalized to the expression of the endogenous control Hs03927097_g1 GAPDH (Applied Biosystems). Relative fold-changes in TERT gene expression were determined by the $\triangle \triangle C T$ method [24]. Shelterin complex TRF1, TRF2, POT1, TPP1, TIN2, and POT1 expression were also measured by qRT-PCR. The PCR conditions with the primer sequences for the six shelterin components are shown in Table S4. All qRT-PCR assays were performed and analyzed on the ABI ViiA 7 Real-Time PCR System (Applied Biosystems).

\subsection{Telomere Length Measurement}

After gDNA extraction, telomere lengths were measured using Absolute Human Telomere Length Quantification qPCR Assay Kit (AHTLQ, Catalog \#8918; ScienCell Research Laboratories, Carlsbad, CA, USA) according to the manufacturer's instructions, as previously reported [25]. Briefly, a single copy reference primer set was used as a reference for data normalization where it recognizes and amplifies a $100 \mathrm{bp}$-long region on human chromosome 17. A reference genomic DNA sample with known telomere length was used as a reference for the calculation of telomere length of the target samples. All AHTLO assays were performed and analyzed on the CFX96 Touch ${ }^{\mathrm{TM}}$ Real-Time PCR Detection System (Bio-Rad).

\subsection{Statistical Analysis}

All data were expressed as the mean \pm S.D. or median (interquartile range). Student $t$-test or Mann-Whitney $U$ test was used to compare continuous variables, while chi-square test or Fisher's exact test to compare categorical variables. Survival analysis was performed using the Kaplan-Meier method to estimate the cumulative rate, and the difference was evaluated based on the log-rank test. The prognostic factors for overall survival (OS) and time to progression (TTP) were performed using the Cox proportional hazard model with univariate and multivariate analysis. A side-step P-value of less than 0.05 was considered to indicate statistically significant differences. Statistical analyses were performed using IBM SPSS Statistics 20.0 (SPSS Inc., Chicago, IL, USA).

\section{Conclusions}

Our analysis of TERT and telomere alterations demonstrates that the TERT-telomere network has a crucial role in all-stage liver carcinogenesis including the development and progression of HCC, with differential TERT factors involved over HCC stage progression. The present findings highlight the utility of TERT genetic alterations and aberrant telomere biology as excellent candidate biomarkers for early diagnosis and monitoring during treatment. Future larger studies should be conducted to further evaluate whether the TERT 
pathway might serve as a potential therapeutic target and define distinct prognostic classes for HCC.

Supplementary Materials: The following are available online at https:/ / www.mdpi.com/article/10 .3390 / cancers 13092160/s1, Figure S1: Correlations between the expression statuses of the shelterin complex proteins, TERT expression, and telomere length, Figure S2: Relationship between the TERT promoter mutation and the outcome of patients undergoing surgical resection according to the cause of HCC, Table S1: Functional interactions between TERT-interacting genes, Table S2: Correlations between the expression statuses of the shelterin complex proteins, TERT expression, and telomere length, Table S3: Primer and probe design for gene expression, Table S4: PCR conditions with the primer sequences for TRF1, TRF2, POT1, TPP1, TIN2, and RAP1.

Author Contributions: Conceptualization, J.-W.J.; Resources, S.-K.L., H.-C.N., P.-S.S., S.-H.B., J.-Y.C., and S.-K.Y.; Investigation J.-S.K., H.-S.K., C.-M.K., and J.-Y.P.; Data curation, J.-W.J., J.-S.K., H.-S.K., and K.-Y.T.; Writing-original draft, J.-W.J.; Writing-review and editing, J.-S.K., H.-S.K., S.-K.L., H.-C.N., P.-S.S., S.-H.B., J.-Y.C., S.-K.Y., and J.-W.J.; Supervision, J.-W.J. All authors have read and agreed to the published version of the manuscript.

Funding: This study was supported by Basic Science Research Program through the National Research Foundation of Korea (NRF) funded by the Ministry of Science, ICT \& Future Planning (NRF-2019R1A2C1009439). This study was supported by The Research Supporting Program of The Korean Association for the Study of the Liver and The Korean Liver Foundation (KASLKLF2018-02).

Institutional Review Board Statement: The study was conducted according to the guidelines of the Declaration of Helsinki and approved by the Institutional Review Board of Seoul St. Mary's Hospital, Seoul, Korea (KC19SESI0195).

Informed Consent Statement: Informed consent was obtained from all subjects involved in the study.

Data Availability Statement: The data presented in this study are available on request from the corresponding author. Associated clinical data cannot be provided to maintain patient confidentiality.

Acknowledgments: We thank Jae-Won Joh, Department of Surgery, Samsung Medical Center, Sungkyunkwan University School of Medicine and Hee-Jung Wang, Department of Surgery, School of Medicine, Ajou University for providing the surgical tissue samples.

Conflicts of Interest: The authors have no financial or personal relationships with other persons or organizations that could inappropriately affect the work. J.-W.J. has served as a consultant to or has served on the advisory board of BMS, Gilead, AbbVie and Bayer.

$\begin{array}{ll}\text { Abbreviations } \\ \text { HBsAg } & \text { hepatitis B surface antigen } \\ \text { HBV } & \text { hepatitis B virus } \\ \text { CHB } & \text { chronic hepatitis B } \\ \text { HCC } & \text { hepatocellular carcinoma } \\ \text { NGS } & \text { next generation sequencing } \\ \text { OBI } & \text { occult HBV infection } \\ \text { PCR } & \text { polymerase chain reaction } \\ \text { TERT } & \text { telomerase reverse transcriptase } \\ \text { WGS } & \text { whole-genome sequencing }\end{array}$

\section{References}

1. Siegel, R.L.; Miller, K.D.; Jemal, A. Cancer statistics, 2016. CA Cancer J. Clin. 2016, 66, 7-30. [CrossRef] [PubMed]

2. Lee, J.-S. The mutational landscape of hepatocellular carcinoma. Clin. Mol. Hepatol. 2015, 21, 220. [CrossRef] [PubMed]

3. Zucman-Rossi, J.; Villanueva, A.; Nault, J.-C.; Llovet, J.M. Genetic landscape and biomarkers of hepatocellular carcinoma. Gastroenterology 2015, 149, 1226-1239.e1224. [CrossRef] [PubMed]

4. Ma, Z.-X.; Yang, C.-M.; Li, M.-G.; Tu, H. Telomerase reverse transcriptase promoter mutations in hepatocellular carcinogenesis. Hepatoma Res. 2019, 2019. [CrossRef] 
5. Nault, J.C.; Mallet, M.; Pilati, C.; Calderaro, J.; Bioulac-Sage, P.; Laurent, C.; Laurent, A.; Cherqui, D.; Balabaud, C.; Zucman-Rossi, J. High frequency of telomerase reverse-transcriptase promoter somatic mutations in hepatocellular carcinoma and preneoplastic lesions. Nat. Commun. 2013, 4, 1-7. [CrossRef] [PubMed]

6. Nault, J.-C.; Ningarhari, M.; Rebouissou, S.; Zucman-Rossi, J. The role of telomeres and telomerase in cirrhosis and liver cancer. Nat. Rev. Gastroenterol. Hepatol. 2019, 15, 544-558. [CrossRef]

7. Huang, D.-S.; Wang, Z.; He, X.-J.; Diplas, B.H.; Yang, R.; Killela, P.J.; Meng, Q.; Ye, Z.-Y.; Wang, W.; Jiang, X.-T. Recurrent TERT promoter mutations identified in a large-scale study of multiple tumour types are associated with increased TERT expression and telomerase activation. Eur. J. Cancer 2015, 51, 969-976. [CrossRef]

8. Okamoto, K.; Seimiya, H. Revisiting telomere shortening in cancer. Cells 2019, 8, 107. [CrossRef]

9. In der Stroth, L.; Tharehalli, U.; Günes, C.; Lechel, A. Telomeres and Telomerase in the Development of Liver Cancer. Cancers 2020, 12, 2048. [CrossRef]

10. Kawai-Kitahata, F.; Asahina, Y.; Tanaka, S.; Kakinuma, S.; Murakawa, M.; Nitta, S.; Watanabe, T.; Otani, S.; Taniguchi, M.; Goto, F. Comprehensive analyses of mutations and hepatitis B virus integration in hepatocellular carcinoma with clinicopathological features. J. Gastroenterol. 2016, 51, 473-486. [CrossRef]

11. Lee, H.W.; Park, T.I.; Jang, S.Y.; Park, S.Y.; Park, W.-J.; Jung, S.-J.; Lee, J.-H. Clinicopathological characteristics of TERT promoter mutation and telomere length in hepatocellular carcinoma. Medicine 2017, 96, e5766. [CrossRef] [PubMed]

12. Yu, J.I.; Choi, C.; Ha, S.Y.; Park, C.-K.; Kang, S.Y.; Joh, J.-W.; Paik, S.W.; Kim, S.; Kim, M.; Jung, S.H. Clinical importance of TERT overexpression in hepatocellular carcinoma treated with curative surgical resection in HBV endemic area. Sci. Rep. 2017, 7, 1-12. [CrossRef] [PubMed]

13. Ako, S.; Nouso, K.; Kinugasa, H.; Matsushita, H.; Terasawa, H.; Adachi, T.; Wada, N.; Takeuchi, Y.; Mandai, M.; Onishi, H.; et al. Human Telomerase Reverse Transcriptase Gene Promoter Mutation in Serum of Patients with Hepatocellular Carcinoma. Oncology 2020, 98, 311-317. [CrossRef] [PubMed]

14. Li, X.; Xu, W.; Kang, W.; Wong, S.H.; Wang, M.; Zhou, Y.; Fang, X.; Zhang, X.; Yang, H.; Wong, C.H. Genomic analysis of liver cancer unveils novel driver genes and distinct prognostic features. Theranostics 2018, 8, 1740. [CrossRef] [PubMed]

15. Nault, J.C.; Calderaro, J.; Di Tommaso, L.; Balabaud, C.; Zafrani, E.S.; Bioulac-Sage, P.; Roncalli, M.; Zucman-Rossi, J. Telomerase reverse transcriptase promoter mutation is an early somatic genetic alteration in the transformation of premalignant nodules in hepatocellular carcinoma on cirrhosis. Hepatology 2014, 60, 1983-1992. [CrossRef] [PubMed]

16. Pinyol, R.; Tovar, V.; Llovet, J.M. TERT promoter mutations: Gatekeeper and driver of hepatocellular carcinoma. J. Hepatol. 2014, 61, 685-687. [CrossRef] [PubMed]

17. Low, K.C.; Tergaonkar, V. Telomerase: Central regulator of all of the hallmarks of cancer. Trends Biochem. Sci. 2013, 38, 426-434. [CrossRef]

18. Artandi, S.E.; Chang, S.; Lee, S.-L.; Alson, S.; Gottlieb, G.J.; Chin, L.; DePinho, R.A. Telomere dysfunction promotes non-reciprocal translocations and epithelial cancers in mice. Nature 2000, 406, 641-645. [CrossRef]

19. El Idrissi, M.; Hervieu, V.; Merle, P.; Mortreux, F.; Wattel, E. Cause-specific telomere factors deregulation in hepatocellular carcinoma. J. Exp. Clin. Cancer Res. 2013, 32, 64. [CrossRef] [PubMed]

20. Kim, H.; Yoo, J.E.; Cho, J.Y.; Oh, B.K.; Yoon, Y.S.; Han, H.S.; Lee, H.S.; Jang, J.J.; Jeong, S.H.; Kim, J.W.; et al. Telomere length, TERT and shelterin complex proteins in hepatocellular carcinomas expressing "stemness"-related markers. J. Hepatol. $2013,59,746-752$. [CrossRef]

21. Korean Liver Cancer Association; National Cancer Center. 2018 Korean Liver Cancer Association-National Cancer Center Korea practice guidelines for the management of hepatocellular carcinoma. Gut Liver 2019, 13, 227-299. [CrossRef] [PubMed]

22. Edmondson, H.A.; Steiner, P.E. Primary carcinoma of the liver. A study of 100 cases among 48,900 necropsies. Cancer 1954, 7, 462-503. [CrossRef]

23. Park, I.J.; Yu, Y.S.; Mustafa, B.; Park, J.Y.; Seo, Y.B.; Kim, G.D.; Kim, J.; Kim, C.M.; Noh, H.D.; Hong, S.M.; et al. A Nine-Gene Signature for Predicting the Response to Preoperative Chemoradiotherapy in Patients with Locally Advanced Rectal Cancer. Cancers 2020, 12, 800. [CrossRef] [PubMed]

24. Livak, K.J.; Schmittgen, T.D. Analysis of relative gene expression data using real-time quantitative PCR and the $2-\Delta \Delta C T$ method. Methods 2001, 25, 402-408. [CrossRef] [PubMed]

25. O'Callaghan, N.; Dhillon, V.; Thomas, P.; Fenech, M. A quantitative real-time PCR method for absolute telomere length. Biotechniques 2008, 44, 807-809. [CrossRef] [PubMed] 